\title{
Dynamic Performance Analysis of Cogeneration Potential in Photovoltaic Power System.
}

\author{
O. S. Babalola $^{1^{*}} \quad$ T. O. Ajewole ${ }^{2} \quad$ O. A. Komolafe ${ }^{1^{*}}$ \\ 1. Department of Electronic and Electrical Engineering, Obafemi Awolowo University, Ile-Ife. Nigeria \\ 2. Department of Electronic and Electrical Engineering, Osun State University, Oshogbo, Nigeria
}

\begin{abstract}
The solar cell junction is susceptible to performance degradation, due to internal temperature rise which is further perturbed by ambient temperature rise. The conversion efficiency of the solar module remarkably drops because of temperature rise. The diurnal module temperature hovers above 60 degrees $\mathrm{C}$. It is possible to keep the conversion efficiency high by water-cooled or air blast equipment. This project is a preliminary study of the impact of temperature variation across seasons on the dynamic performance of photovoltaic power system. The photovoltaic (PV) module was mounted on a test rig at latitude 7 degree on top of physics building Obafemi Awolowo University campus. The output voltage of the PV module was applied to a resistive load and system efficiency performance measured over a period of time covering the dry and rainy seasons. Measurements of ambient temperature, PV junction operating temperature, wind speed, solar irradiance, and power delivered were carried out. A numerical algorithm was developed to analyse the data. The transport medium can store heat energy from the solar module, so that a large amount of hot water can be produced. The results show that this thermal transfer benefit, positions the PV system in good stead, for Microgrid: Cogeneration Energy Efficiency functionality.
\end{abstract}

Keywords: Conversion efficiency, Photovoltaic power system, PV module, Water-cooled equipment, Cogeneration.

\section{Introduction}

Solar power could be the leading source of electricity if efficient harvesting of adequate amount of the energy delivered to the earth from the sun on a daily basis is possible. On the equator at noon $1000 \mathrm{~W} / \mathrm{m}^{2}$ of sun energy touches the ground. Unfortunately only about 20 percent of this power can be transferred into usable energy (Anderson et al 2003). This inefficiency is directly related to the percentage of photons that are absorbed when sunlight impinges on the PV surface. All electromagnetic radiation, including sunlight, is composed of particles called photons, which carry specific amounts of energy determined by the spectral properties of their source. Photons propagate with wavelength $\lambda$, being related to the photon energy by

$E_{\lambda}=\frac{h c}{\lambda}$

where $\mathrm{h}$ is plank's constant and $\mathrm{c}$ is the speed of light. Only photons with sufficient energy to create an electron-hole pair will contribute to the energy conversion process. Photons with energy greater than $1.1 \mathrm{eV}$ can generate electron-hole pairs (Burgers, 2005). Thus, the spectral nature of sunlight is an important consideration in the performance of solar cells (Gray, 2003).

The physics of the PV cell is very similar to the classical p-n junction diode. When light is absorbed by the junction, the energy of the absorbed photons is transferred to the electron system of the material, resulting in the creation of charge carriers that are separated at the junction. The charge carriers may be electron-ion pairs in a liquid electrolyte, or electron-hole pairs in a solid semiconducting material. The charge carriers in the junction region create a potential gradient, get accelerated under the electric field and circulate as the current through an external circuit. The square of the current times the resistance of the circuit is the power converted into electricity. The remaining power of the photon elevates the temperature of the cell (Kluftinger, 2000). It is known that the conversion efficiency of the solar module remarkably drops because of temperature rise. The diurnal module temperature hovers above 60 degrees $\mathrm{C}$ (Fujii, et al, 2013). It is possible to keep the conversion efficiency high by water-cooled or air blast equipment. The benefits of cooling on heightened temperature is primarily efficiency gain and it is immediate and soothing to the mass material (composite) from which the PV is fabricated. This culminates in reduced thermal stress and consequently long life of system device. The accompanying energy gain retrievable from the underpinning storage device/transport medium, supplies useful heat energy needs. This project is a preliminary study of the impact of temperature variation, across seasons, on the dynamic performance of photovoltaic power system.

\section{Methodology}

The photovoltaic (PV) module was mounted on a test rig at latitude 7 degree south facing on top of physics building Obafemi Awolowo University campus. The following data were acquired using the Campbell Scientific 
application package-Loggernet: ambient temperature, irradiance, voltage across resistive load and PV junction temperature. This was done daily in 2-minute interval for one year. Shaded pyranometer, optical thermometer, and anemometer, were used for measurement of irradiance, temperature and wind speed respectively. The power generated by the PV array was computed using irradiance (GG), nominal operating cell temperature (NOCT), open circuit voltage $\left(\mathrm{V}_{\mathrm{OC}}\right)$, short circuit current $\left(\mathrm{I}_{\mathrm{SC}}\right)$. The last three were obtained from the manufacturer's specification for the PV. The measured data for photovoltaic cell junction, ambient temperature, irradiance and load voltage are provided as daily average. The output voltage of the PV module was applied to a resistive load and system efficiency performance determined over a period of time covering the dry and rainy seasons. A numerical algorithm was developed to analyse the data.

The actual output of a PV module or system in the field is a function of orientation, total irradiance, air temperature, soiling and various system-related losses (Angelopoulos, 2004). The PV conversion efficiency $\left(\eta_{C}\right)$ is calculated from the measured maximum or peak PV power $P_{\max }$, device area (A), and total incident irradiance $\left(G_{i}\right)$ :

$\eta_{C}=\frac{P_{\max }}{G_{i} A} 100 \%$

The PV conversion efficiency $\eta_{c}$ is inversely proportional to the area definition used in equation (2). For the PV modules, an aperture-area definition was used. The aperture-area definition is the total area minus the frame area. Even though the power or peak watt rating on the module's name plate is often given with reference to Standard Test Condition (STC), the prevailing conditions under natural light do not commonly match name plate conditions. The manufacturer assigned name plate ratings to a given module model number is usually higher than the measured power output in the field. Module typically runs at $40^{\circ}$ to $60^{\circ} \mathrm{C}$, if the name plate rating is determined at $25^{\circ} \mathrm{C}$, then the actual power produced is less. The temperature coefficient for the peak power is usually negative. The nominal operating cell temperature (NOCT) is a rating designed to give information about the thermal qualities of a module and a more realistic estimate of the power in the field on a clear sky sunny day, at solar noon. The NOCT of a module is a fixed temperature the module would operate at when exposed to the nominal thermal environment $\left(20^{\circ} \mathrm{C}\right.$ air temperature, $800 \mathrm{~W} / \mathrm{m}^{2}$ total irradiance, and a wind speed of $\left.1 \mathrm{~m} / \mathrm{s}\right)$. The NOCT for the sample module data provides information for the prediction of PV array performance for the site. The installed NOCT is up to $15^{\circ} \mathrm{C}$ warmer for roof-mounted applications than a free-standing module depending on the stand-off distance between the module and the roof. The temperature can be calculated from the NOCT provided by the manufacturer or measured values of NOCT and air temperature using (Emery, 2003),

$T_{C}=T_{a m b}+\left(N O C T-20^{\circ} C\right) \frac{G_{i}}{800}$

where $T_{a m b}$, is ambient temperature, ${ }^{\circ} \mathrm{C} ; T_{C}$ is cell temperature, ${ }^{\circ} \mathrm{C}$.

In a dynamic meteorological system for PV module performance measurement, capable of providing instantaneous efficiency figures, the diurnal variation in irradiance and ambient (air) temperature aggregate over a period of days and/or months to delineate seasons. PV module efficiency is particularly affected by sunlight intensity (irradiance) and temperature, which vary due to the daily sunrise-sunset cycle, and seasonal sun trajectories at varying latitudes (Moghbelli, 2006).

\subsection{Experimental set up}

The schematic in figure 1 was set up with $66 \Omega 18 \mathrm{~W}$ resistor as load. Observations were made in 2-minute intervals for the seasons of the year. The data acquisition system captured the ambient temperature, solar radiation, PV junction temperature, load current and wind speed. The system efficiency performances during "hot and dry", "wet", and "hot", seasons were observed. The electrical energy delivered by the photovoltaic system to the load was computed by summing the 2-minute stored values. The system efficiency of the solar photovoltaic supply was computed using:

$\eta_{s}=\frac{\int_{\tau_{1}}^{\tau_{2}} P d \tau}{A \int_{\tau_{1}}^{\tau_{2}} G_{i} d \tau}$

where,

A is PVM representative area, $\mathrm{m}^{2}$; 
$G_{i}$ is the global incident irradiance, $\mathrm{W} / \mathrm{m}^{2}$;

$\mathrm{P}$ is the electrical power delivered to the load, $\mathrm{W}$;

$\tau_{1}$ and $\tau_{2}$ are the beginning and end of observation duration.

\section{Results and Analysis}

The test results and analysis were carried out using MATLAB as a tool, the performance curves were constructed, with the measurements taken as data inputs. The performance of the PV module in terms of efficiency over seasons is presented in graphs, for the different seasons with irradiance/temperature curve overlay.

\subsection{Temperature effects in dynamic PV system}

The solar cell junction is susceptible to performance degradation, due to internal temperature rise which is further perturbed by ambient temperature rise. The junction temperature (monitored by sensing the back temperature of the PV module) of the prototype was monitored by optical temperature sensor; the operating temperature variation over seasons is shown in figure 2. The efficiency ascent in the November/December region is curious, however, the PV junction temperature curve overlaid on the efficiency plot reveals the impact of the former on the latter. It reveals the effect of uninhibited penetration of irradiance coupled with the characteristic cool breeze of the less humid "hot/dry" region. The growing double arrows within the ellipse, highlights the ascent of efficiency with the advent of PV junction temperature diminution.

The weather of the days covered by the ellipse characteristically differentiates between the "hot/dry" and the "hot" region. This phenomenon translates directly to initial cost reduction in PV systems because more power is harvested when efficiency is higher and reduced number of PVs will be required to support the same load. Figure 3 shows that ambient temperature in the "hot" region is more severe than in the "hot/dry" region. The blow up plot of the ellipse portion in figure 4 further elucidates this fact.

\subsection{Seasonal variations}

Solar energy is both variable and intermittent in its distribution; solar power output is variable in that it produces different amounts of power at different times of the day, and on different days. However, this patterned variability forms the basis for its relative predictability (Goetzberger, and Hoffmann, 2005), see figure 5. The solution to solar power supply challenges is further elucidated by the understanding of its intermittence, as every night, solar photovoltaic systems do not produce power, unless by the release of power storage provision that may accompany the system. Even though earth-sun relational movement is the causative agent of seasonal variations, solar intensity (irradiance) is the parameter whose level or absence translates directly to these seasonal variations in PV systems' performance. This is shown in the efficiency versus time of day plot of figure 6. The other parameter like a Siamese twin to irradiance is temperature. The heating effect of the late morning flux of solar energy usually manifests about two hours later (Geiger, 1950). The efficiency performance of the PV module is remarkably enhanced around the clement temperature hours of the day, with turning points observable at $10.00 \mathrm{am}$ and $3.00 \mathrm{pm}$.

\subsubsection{Irradiance effect on efficiency}

The best efficiency window spanning $9.30 \mathrm{am}$ and $4.00 \mathrm{pm}$ includes the period of the day when irradiance is at its zenith. Figure 7 shows the overlay of daily hourly irradiance curve on efficiency performance of the PV with variable load, using average hourly irradiance and efficiency. Even though the efficiency appears to capsize in this portion, it is by far the most stable efficiency period. This effect is the repercussion of increased solar flux that peaks at 12 noon daily. Back cooling of the device will greatly enhance the efficiency performance in this region of very high insolation.

\subsubsection{Annual seasonal variability of PV system efficiency}

It is possible to observe three different solar energy segment for "hot/dry", "hot", and "wet" seasons corresponding to November/December, February/March, and July/August, in that order. Figure 8 shows the trend in the efficiency performance of the PV, with the overlay of irradiance curve, it is easy to see that even though there is dearth in the supply of irradiance in the period covering July/August, the system efficiency in that period clearly towers above that of February/March, which actually has adequate irradiance but inclement average daily temperatures. It is interesting to note the relative stability on both sides of July/August in efficiency. The region following July/August is characterized by adequate irradiance without cloud overcast and temperature is less regular at its upper threshold. Making sure that installation is cognisant of professional best practices (Risser, 1995), a heat exchanger incorporation to the PV that is PV/T will ensure efficiency imitates the accent of the daily irradiance profile. 


\section{Conclusion}

The diminution in efficiency performance of PV module is further aggravated by heightened device junction temperature. The instantaneous, overwhelmingly high efficiency in wet season is as a result of the sporadic showers and coolness associated with the season. Observably, the rising efficiency profile from 10am can be steadied by cooling, maintaining an all-time higher value than without cooling, as long as there is irradiance. As a result of the temperature gap of 20-60 degree $\mathrm{C}$, the prospect of hot water harvesting using the PV in cogeneration mode is realizable. The transport medium can store heat energy from the solar module, so that a large amount of hot water can be produced, however a heat pump may be a desirable addendum for cool wet season, if heat energy is required, as the case may be. The results show that this thermal transfer benefit positions the PV System in good stead, for Microgrid: Cogeneration Energy Efficiency functionality.

One possible research direction is a programmed pumping device acting on two temperature thresholds of 25 and 30 degrees $\mathrm{C}$ for cut-off and cut-in respectively for a PV/T. Also, heat pump should be incorporated to cater for heat energy needs in wet season.

\section{Acknowledgement}

The atmospheric physics unit of physics department of Obafemi Awolowo University provided the Campbell Scientific application package-Loggernet for the meteorological data acquisition.

\section{References}

Anderson, E., Dohan, C., Sikora, A., Bitar, S. J. and McNeill, J. A., (2003). Solar Panel Peak Power Tracking System. Bachelor of Science Thesis. Worcester Polytechnic Institute.

Angelopoulos, K., (2004). Integration of Distributed Generation in Low Voltage Networks: Power Quality and Economics. pp.18

Burgers, A.R., (2005). New Metallisation Patterns and Analysis of Light Trapping for Silicon Solar Cells.

Emery, K., (2003). Measurement and Characterization of Solar Cells and Modules National Renewable Energy Laboratory, Golden, CO, USA

Geiger, R., (1950). The Climate near The Ground. A Translation of the Second German Edition. Harvard University Press Cambridge, Massachusettes

Goetzberger, A., and Hoffmann,V. U.,(2005).Photovoltaic Solar Energy Generation. Springer Series in Optical Sciences Publisher, pp.3-2

Gray, J. L., (2003). The Physics of the Solar Cell. Handbook of Photovoltaic Science and Engineering.

Kluftinger, B. G. (2000). An experimental study of the quasi-Fermi level separations in quantum well solar cells. $\mathrm{Ph} . \mathrm{D}$. Thesis, University of London Imperial College of Science, Technology and Medicine. London

Fujii, M., Yanagihara, H., Mitsumoto, S., Kikugawa, S., Tokoro, T., Fukuma, M., Improvement Conversion Efficiency through Water-cooled Equipment in Photovoltaic System, Journal of International Council on Electrical Engineering Vol. 3, No. 1, pp.97 101, 2013

Moghbelli, H. and Vartanian, R., (2006). Implementation of the Movable Photovoltaic Array to Increase Output Power of the Solar Cells, Proceedings of the International Conference on Renewable Energy for Developing Countries.

Risser, V., (1995) Stand-Alone Photovoltaic System -A handbook of recommended design practices. Daystar, Inc., Las Cruces, New Mexico. 


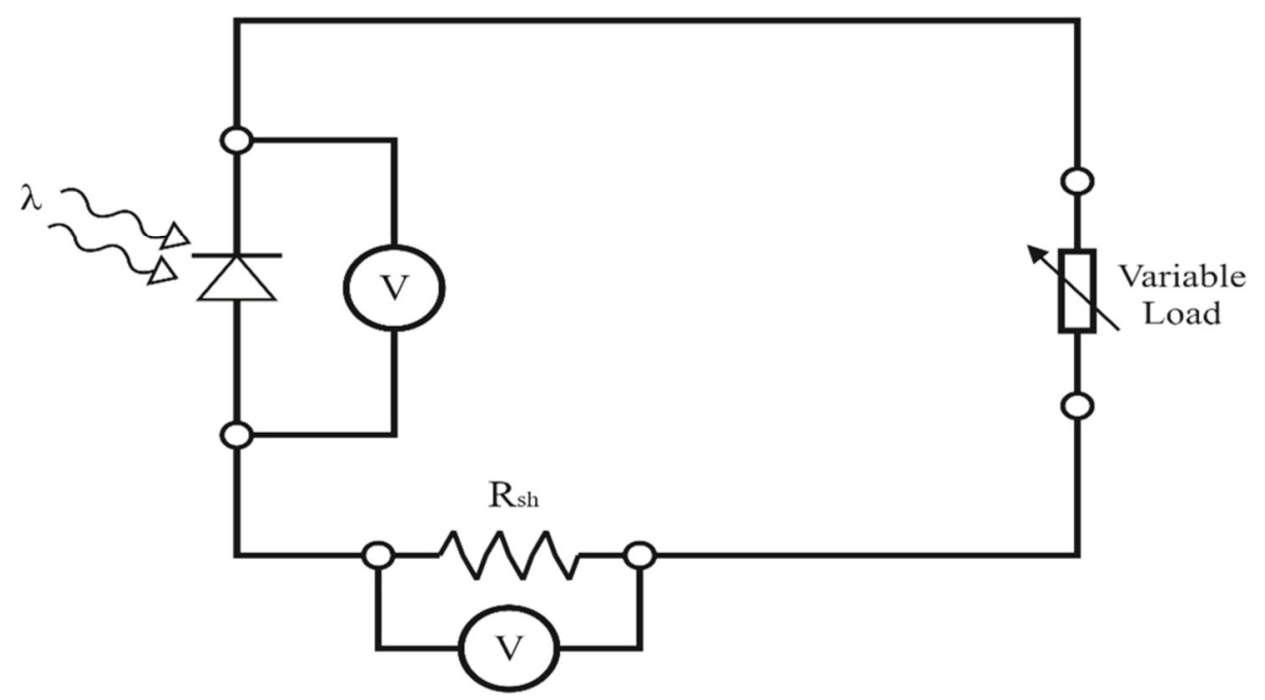

Figure 1: PVM variable load schematic

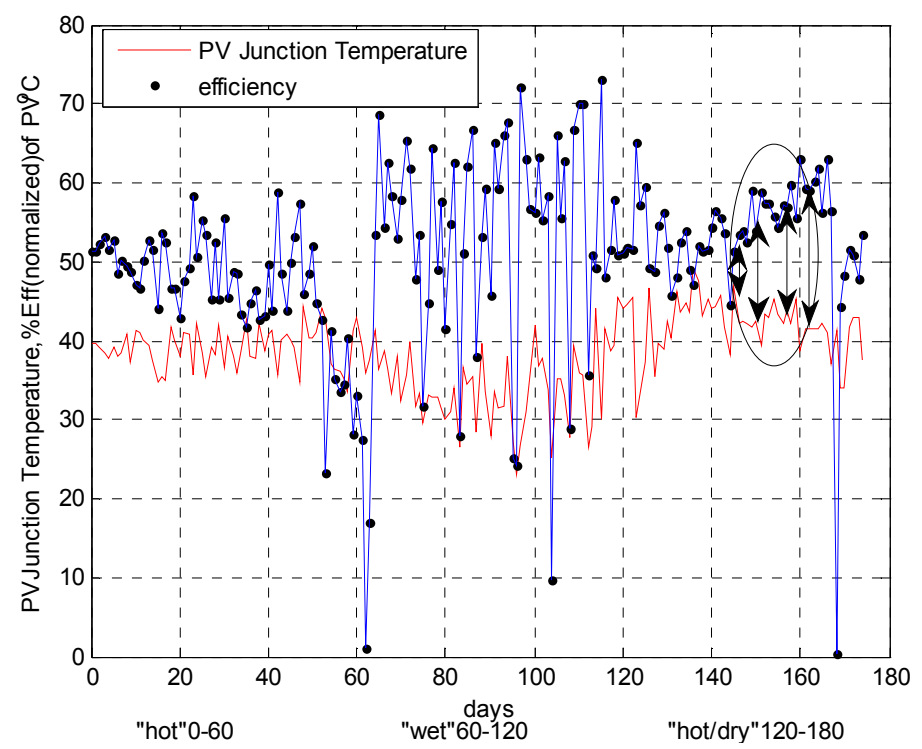

Figure 2: Effect of PV junction temperature on efficiency (The growing double arrow within the ellipse highlights the ascent of efficiency with the advent of PV junction temperature diminution). 


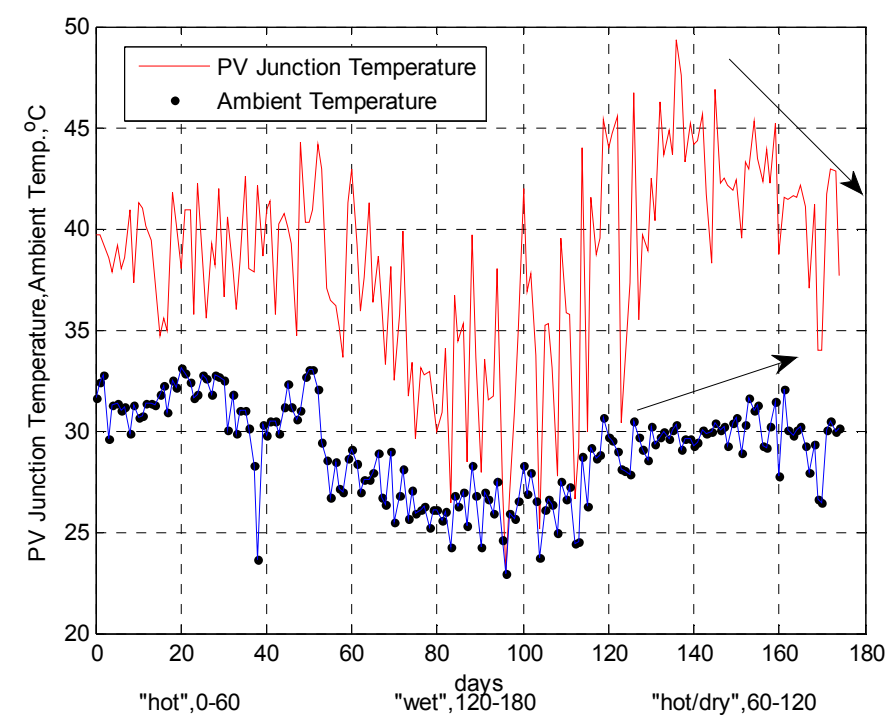

Figure 3: Ambient and PV junction temperature curves overlay. (The ascent and descent arrows highlight the prevalent trend in the region).

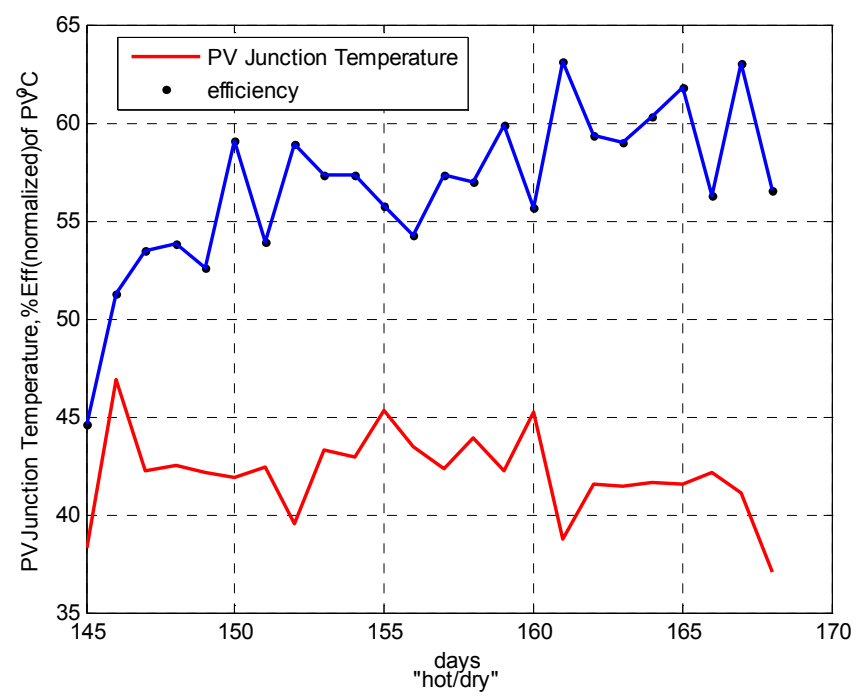

Figure 4: Blow up plot of ellipse portion of figure 2 


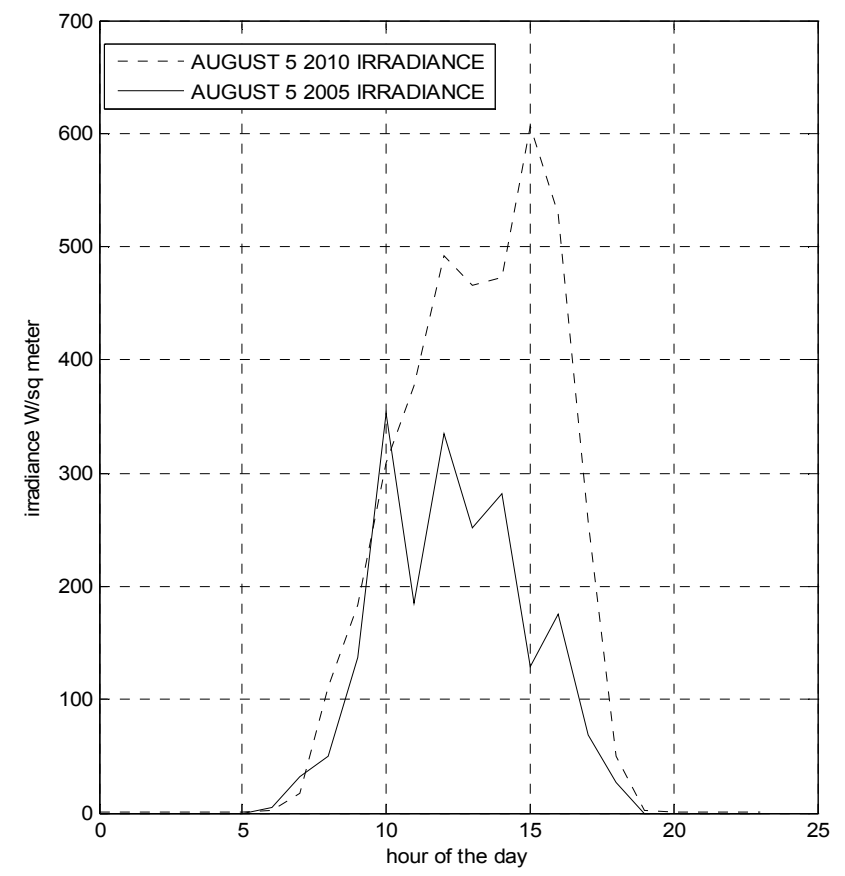

Figure 5: Irradiance intermittence and variability curve

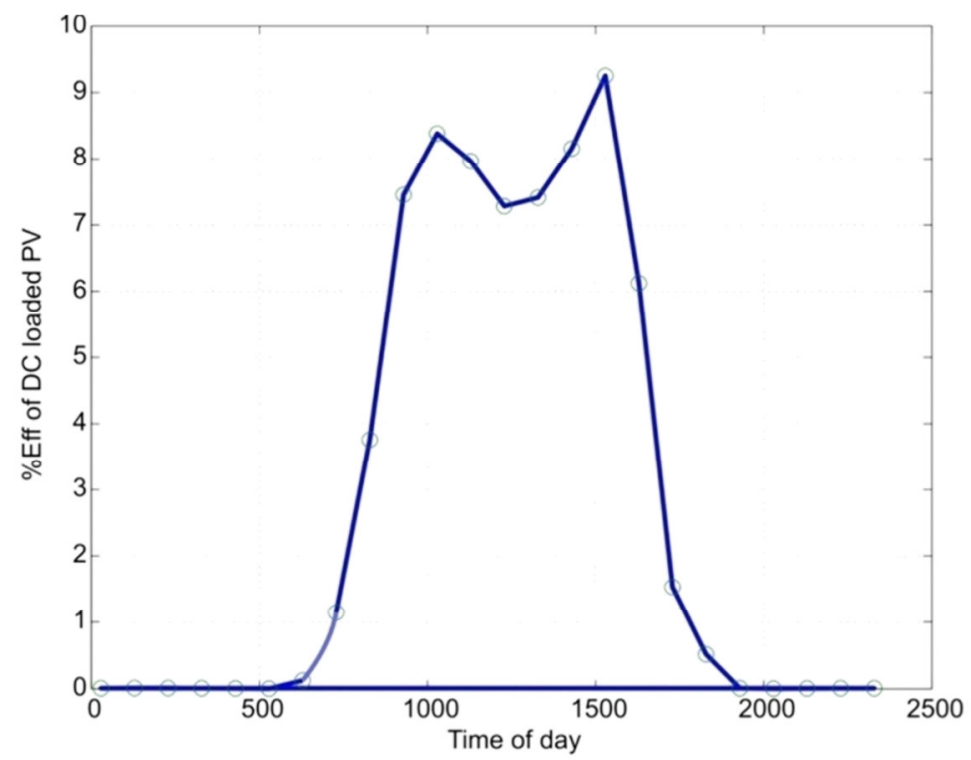

Figure 6: Efficiency versus time of day plot 


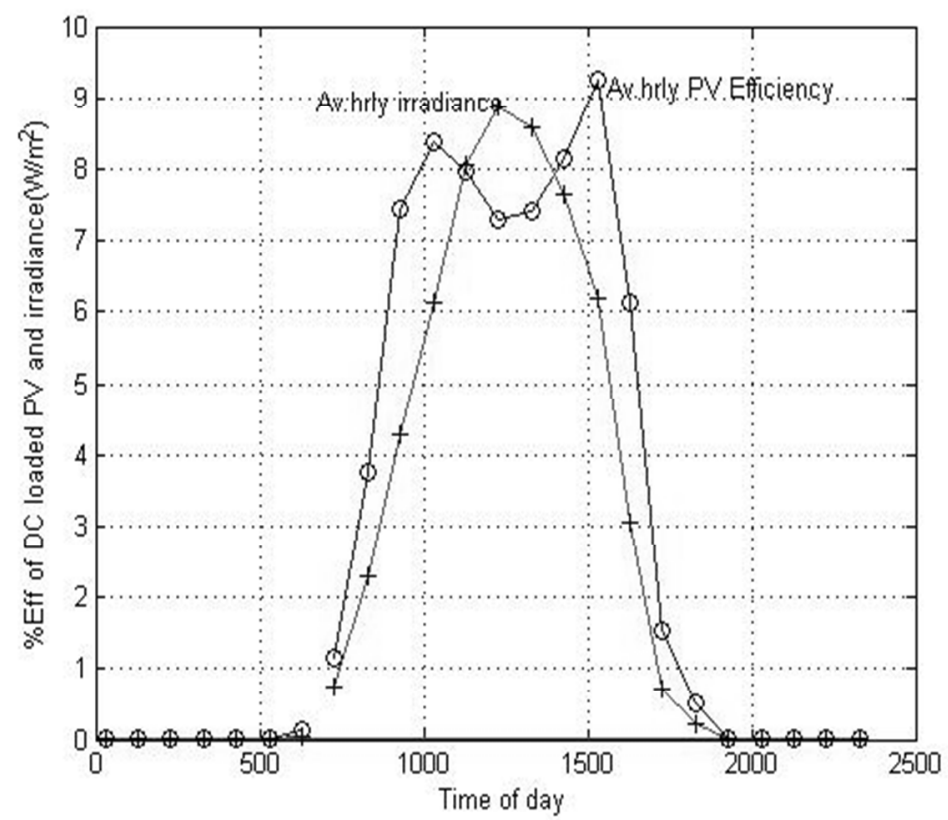

Figure 7: Overlay of daily average irradiance curve on efficiency performance of the PV on DC load, using average hourly irradiance and efficiency.

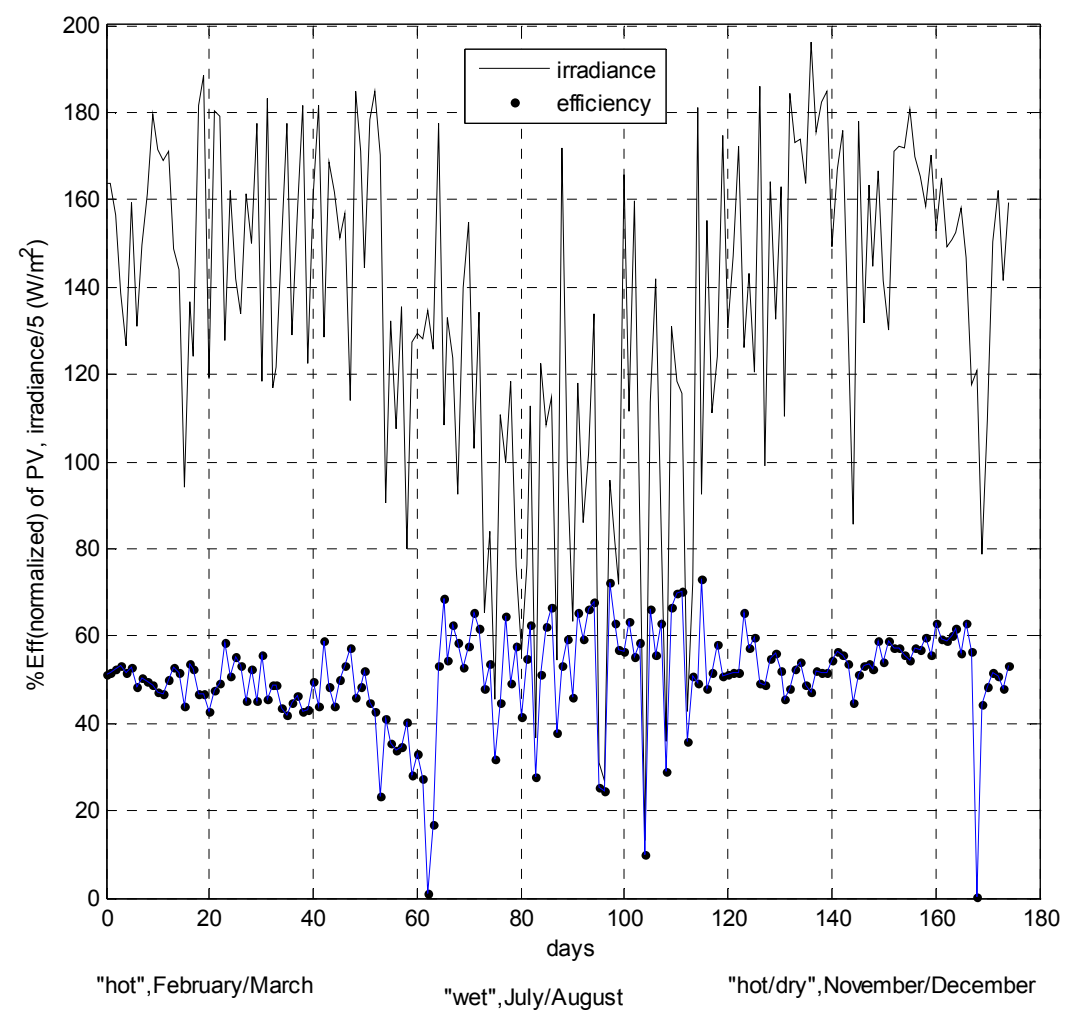

Figure 8: Annual trend in seasonal PV System efficiency/irradiance 